\title{
Dynamic classification of escape time Sierpiński curve Julia sets
}

by

\author{
Robert L. Devaney (Boston, MA) and \\ Kevin M. Pilgrim (Bloomington, IN)
}

\begin{abstract}
For $n \geq 2$, the family of rational maps $F_{\lambda}(z)=z^{n}+\lambda / z^{n}$ contains a countably infinite set of parameter values for which all critical orbits eventually land after some number $\kappa$ of iterations on the point at infinity. The Julia sets of such maps are Sierpiński curves if $\kappa \geq 3$. We show that two such maps are topologically conjugate on their Julia sets if and only if they are Möbius or anti-Möbius conjugate, and we give a precise count of the number of topological conjugacy classes as a function of $n$ and $\kappa$.
\end{abstract}

1. Introduction. Our goal in this paper is to consider the dynamics of families of rational maps of the form

$$
F_{\lambda}(z)=z^{n}+\frac{\lambda}{z^{n}}
$$

where $\lambda \neq 0$ is a complex parameter and $n \geq 2$. These families have been shown to have a number of interesting dynamical and topological properties, including a variety of different ways that Sierpiński curves arise as the Julia sets for these maps. In this paper we present a dynamic classification of what we call the escape time Sierpiński curve Julia sets in these families.

To describe these sets, we first compute that each of these maps has $2 n$ "free" simple critical points $c_{\lambda}$ given by $c_{\lambda}^{2 n}=\lambda$, but only two critical values given by $v_{\lambda}= \pm 2 \sqrt{\lambda}$. However, like the well-studied quadratic family $Q_{c}(z)=z^{2}+c$, each map has essentially only one free critical orbit since the two critical values map to the same point if $n$ is even, while their orbits are arranged symmetrically with respect to $z \mapsto-z$ if $n$ is odd.

As another similarity with the quadratic family, the point at infinity is a superattracting fixed point for each $\lambda$ when $n>1$, and so it may be the case that the critical orbits enter the immediate basin of this fixed point. We denote the immediate basin of attraction of $\infty$ by $B_{\lambda}$. Since 0 is a pole, there 
is a neighborhood of 0 that is mapped into $B_{\lambda}$. Now either this neighborhood is itself contained in $B_{\lambda}$, or else 0 lies in a disjoint preimage of $B_{\lambda}$, in which case we denote this preimage by $T_{\lambda}$. In the latter case, note that $F_{\lambda}$ maps $T_{\lambda}$ in $n$-to- 1 fashion onto $B_{\lambda}$ while $F_{\lambda} \mid B_{\lambda}$ is also $n$-to- 1 . Hence the only preimages of $B_{\lambda}$ are $B_{\lambda}$ itself and $T_{\lambda}$. Thus, the only way a critical orbit can eventually enter $B_{\lambda}$ is by passing through $T_{\lambda}$. For this reason we call $T_{\lambda}$ the trap door. The following Theorem describes the Julia sets that result when the critical orbits eventually enter $B_{\lambda}$ (see [8]):

TheOrem (Escaping critical orbits). For the family of functions

$$
F_{\lambda}(z)=z^{n}+\frac{\lambda}{z^{n}}
$$

with $n \geq 2$ and nonzero $\lambda \in \mathbb{C}$, if the critical values lie in some preimage of $T_{\lambda}$ (that is, they escape), then the Julia set is a Sierpinski curve.

We remark that if the critical values lie in $B_{\lambda}$ or $T_{\lambda}$, then the Julia sets are very different topologically. If the critical values lie in $B_{\lambda}$, the Julia set is a Cantor set; if they lie in $T_{\lambda}$, the Julia set is a Cantor set of simple closed curves. See [8], [12].

A Sierpinski curve is a planar set that is characterized by the following five properties: the set is compact, connected, locally connected, nowhere dense, and all of the complementary domains are bounded by simple closed curves that are pairwise disjoint. It is known from work of Whyburn [16] that any two Sierpiński curves are homeomorphic. In fact, they are homeomorphic to the well-known Sierpiński carpet fractal. Furthermore, it is known [10] that any homeomorphism between a pair of Sierpiński curves extends to a homeomorphism defined on the entire Riemann sphere. This implies that if two Sierpiński curves are already embedded in the complex plane, then one can speak unambiguously about a homeomorphism between the two being orientation preserving/reversing. The first example of a Sierpiński curve Julia set was given by Milnor and Tan Lei [13].

Let $J\left(F_{\lambda}\right)$ denote the Julia set of $F_{\lambda}$. We call a Julia set for which the critical orbit eventually escapes to $\infty$ an escape time Sierpinski curve and denote by $\kappa=\kappa(\lambda)$ the number of iterations of $F_{\lambda}$ that it takes for the critical orbit to enter the immediate basin of $\infty$. By the previous theorem, we thus need $\kappa \geq 3$ for $J\left(F_{\lambda}\right)$ to be an escape time Sierpiński curve.

It is known [4] that for every $n \geq 2$, the set of parameter values $\lambda$ for which the critical points escape is an open set with infinitely many connected components. For example, in Figure 1, we display the parameter plane for the case $n=2$. Each of the bounded grey regions in this figure contains parameters for which the critical orbits enter $B_{\lambda}$ after three or more iterations and hence the Julia set is a Sierpiński curve. We call these regions in the parameter plane Sierpiński holes. 


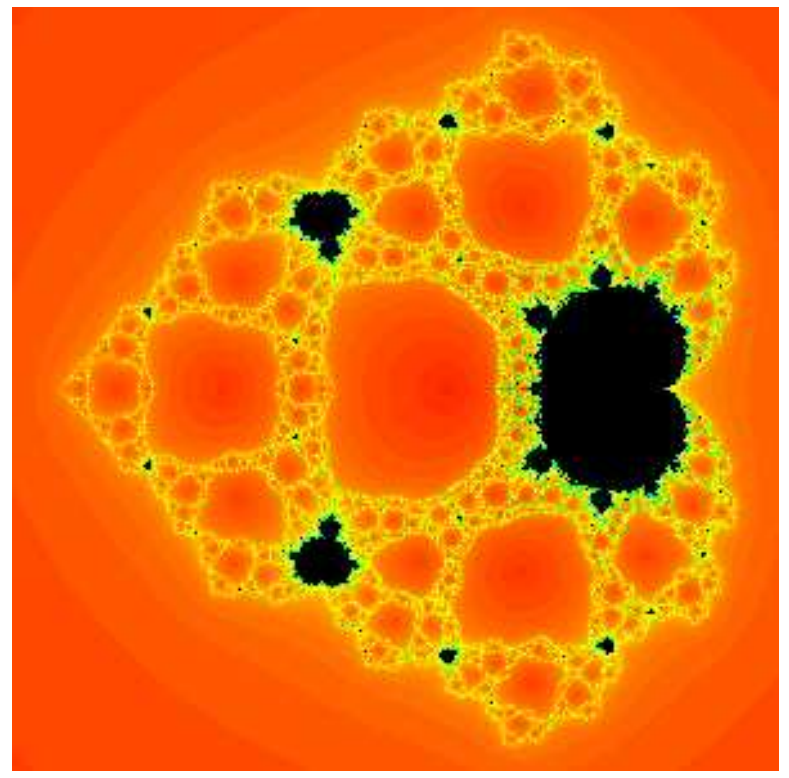

Fig. 1. The parameter plane for the family $z^{2}+\lambda / z^{2}$

In Figure 2 we display the parameter plane for the case $n=3$. All of the bounded grey regions in this case except the small "disk" in the center are Sierpiński holes. In this picture, the central region is the McMullen domain

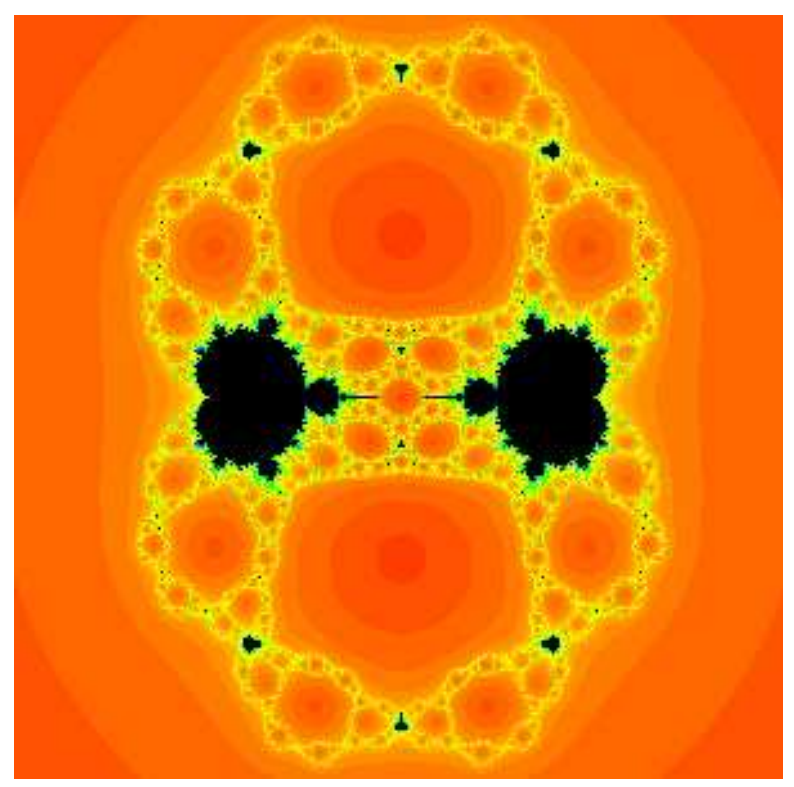

Fig. 2. The parameter plane for the family $z^{3}+\lambda / z^{3}$ 
(see [6], [12]) i.e., the region where the critical values lie in the trap door so the Julia set is a Cantor set of simple closed curves. In both of these figures, the unbounded grey region contains the parameters where the Julia set is a Cantor set. We call this subset of the parameter plane the escape locus.

There are several facts known about the properties of the Sierpiński holes in the parameter plane. The following Theorem was proved in [4] and [14].

TheOREM (Structure of Sierpiński holes). There is a unique center of each Sierpinski hole in the parameter plane, i.e., a parameter for which the critical orbits all land on $\infty$ at the escape time iteration $\kappa$. Also, there are exactly $(n-1)(2 n)^{\kappa-3}$ Sierpiński holes with escape time $\kappa$.

As a consequence, the Julia sets corresponding to parameters drawn from the same Sierpiński hole are always homeomorphic. So the question is: when are the two maps corresponding to these parameters topologically conjugate on their Julia sets? Our first result in this paper is:

Theorem (Escape time conjugacy). Let

$$
F_{\lambda}(z)=z^{n}+\frac{\lambda}{z^{n}} \quad \text { and } \quad F_{\mu}(z)=z^{n}+\frac{\mu}{z^{n}}
$$

where $\lambda$ and $\mu$ are parameters that lie in Sierpinski holes.

(1) If $\lambda$ and $\mu$ lie in the same Sierpinski hole, then $F_{\lambda}$ and $F_{\mu}$ are topologically conjugate on their Julia sets.

(2) If $\lambda$ and $\mu$ lie in Sierpinski holes with different escape times, then $F_{\lambda}$ and $F_{\mu}$ are not topologically conjugate on their Julia sets.

(3) Suppose $\lambda$ and $\mu$ are centers of different Sierpinski holes that have the same escape time. Let $\alpha$ be a primitive $(n-1)$ st root of unity. Then $F_{\lambda}$ and $F_{\mu}$ are topologically conjugate on their Julia sets if and only if, for some integer $j$,

- $\mu=\alpha^{2 j} \lambda$, or

- $\mu=\alpha^{2 j} \bar{\lambda}$.

Therefore, if $\lambda$ and $\mu$ are parameters lying in different Sierpinski holes whose escape times are the same, then $F_{\lambda}$ and $F_{\mu}$ are topologically conjugate on their Julia sets if and only if the centers of these Sierpinski holes have the above property.

This result allows us to give a precise count of the number of different conjugacy classes of escape time Sierpiński curves. In Sections 3 and 4 we shall prove:

THEOREM (Number of conjugacy classes). The number of topological conjugacy classes of escape time Sierpinski curve Julia sets with escape time $\kappa$ is given by

(1) $(2 n)^{\kappa-3}$ if $n$ is odd;

(2) $(2 n)^{\kappa-3} / 2+2^{\kappa-4}$ if $n$ is even. 
For example, when $n=3$, there are exactly $2 \cdot 6^{3}=432$ Sierpiński holes in this family with escape time 6 and exactly 216 different conjugacy classes of such maps. Similarly, there are 120,932,352 Sierpiński holes with escape time 13 and 60,466,176 different conjugacy classes, so clearly there is a great variety of different dynamical behaviors on these escape time Sierpiński curve Julia sets.

REMARKs. 1. The above count of conjugacy classes may be the first of its kind. To the best of our knowledge, for quadratic polynomials for which the critical point is eventually periodic with preperiod $m$ and period $k$, the function of $m$ and $k$ giving the number of topological conjugacy classes on the Julia set is unknown.

2. If $\lambda, \mu$ are centers of Sierpiński holes, then their Julia sets are both Sierpiński curves and so they are homeomorphic. It would be interesting to have a classification of such Sierpiński curves up to a quasiconformal homeomorphism. By a theorem of Bonk ([1, Theorem 7.5]), for any such $J\left(F_{\lambda}\right)$, there is a quasiconformal homeomorphism $h: J\left(F_{\lambda}\right) \rightarrow Y_{\lambda}$, where $Y_{\lambda}$ is a Sierpiński curve in the sphere having area zero and whose complementary domains are round disks. A theorem of Bonk, Kleiner, and Merenkov ([2] and [1, Theorem 7.3]) implies that a quasiconformal map $g: Y \rightarrow Y^{\prime}$ between two such round Sierpiński curves is a Möbius transformation. Therefore, the round Sierpiński curve $Y_{\lambda}$ and the quasiconformal homeomorphism $h$ are unique, up to Möbius transformations. As of this writing, we do not have a concrete example of a pair of centers $\lambda, \mu$ corresponding to nonconjugate maps for which we can prove either that $J\left(F_{\lambda}\right)$ and $J\left(F_{\mu}\right)$ are quasiconformally homeomorphic, or that they are not.

2. Conjugacy results. Our goal in this section is to prove the escape time conjugacy theorem. Let

$$
F_{\lambda}(z)=z^{n}+\frac{\lambda}{z^{n}}
$$

with the complex parameter $\lambda \neq 0$ and $n \geq 2$. Recall that the point at $\infty$ is a superattracting fixed point and we have the immediate basin of attraction of $\infty$ denoted by $B_{\lambda}$. The origin is also a critical point and a pole, and, provided that $|\lambda|$ is small enough, we have the trap door $T_{\lambda}$ which surrounds the origin and is disjoint from $B_{\lambda}$. Each of $B_{\lambda}$ and $T_{\lambda}$ is an open disk that is mapped $n$-to- 1 onto $B_{\lambda}$. The other critical points are the free critical points; they are given by $c_{\lambda}^{2 n}=\lambda$. The critical values are given by $v_{\lambda}= \pm \sqrt{\lambda}$.

Let $\omega$ be a primitive $2 n$th root of unity. Then the Julia set of $F_{\lambda}$, denoted by $J\left(F_{\lambda}\right)$, is symmetric under the map $z \mapsto \omega z$. To see this, note that

$$
F_{\lambda}(\omega z)=\omega^{n} z^{n}+\frac{\lambda}{\omega^{n} z^{n}}=-F_{\lambda}(z)
$$


Thus, if $n$ is even, $F_{\lambda}^{2}(\omega z)=F_{\lambda}^{2}(z)$, where $F_{\lambda}^{2}=F_{\lambda} \circ F_{\lambda}$; if $n$ is odd, the orbits of $F_{\lambda}(\omega z)$ and $F_{\lambda}(z)$ are symmetric under $z \mapsto-z$. Note that this also implies that the orbits of the second images of the free critical points are either the same or symmetric under $z \mapsto-z$ and so all the free critical orbits have the same fate.

We now turn to the conjugacy properties of $F_{\lambda}$. Suppose $F_{\lambda}$ is conjugate to $F_{\mu}$ on $\overline{\mathbb{C}}$ via a Möbius transformation $h$. Since $h$ must map the superattracting fixed point at $\infty$ to itself and the pole at 0 to itself, it follows that $h$ is of the form $h(z)=\omega z$ for some $\omega \in \mathbb{C}$. Then, if $\omega F_{\lambda}(z)=F_{\mu}(\omega z)$, we must have

$$
\omega z^{n}+\frac{\omega \lambda}{z^{n}}=\omega^{n} z^{n}+\frac{\mu}{\omega^{n} z^{n}} .
$$

It follows that $\omega^{n}=\omega$ so that $\omega^{n-1}=1$. Also, we must have $\omega \lambda=\mu / \omega^{n}$, so that $\mu=\omega^{2} \lambda$. Thus any map of the form $F_{\alpha^{2 j} \lambda}$ is Möbius conjugate to $F_{\lambda}$ when $\alpha$ is a primitive $(n-1)$ st root of unity and $j$ is an integer. If $n$ is even, it follows that $F_{\lambda}$ is Möbius conjugate to $F_{\alpha^{j} \lambda}$ for each integer $j$. If $n$ is odd, then the parameters $\alpha^{2 j} \lambda$ all have conjugate dynamics. Similarly, the parameters $\alpha^{2 j+1} \lambda$ have conjugate dynamics, but $F_{\alpha^{2 j} \lambda}$ is not Möbius conjugate to $F_{\alpha^{2 k+1} \lambda}$ for any $j$ and $k$. As an example, when $n=3$, as shown in Figure 2, there are two large Mandelbrot sets along the real axis. When $\lambda$ lies in the main cardioid of the Mandelbrot set on the right, $F_{\lambda}$ has two attracting fixed points. Three of the free critical points are attracted to one of these fixed points, while the other three are attracted to the other fixed point. In the main cardioid in the Mandelbrot set on the left, the map $F_{-\lambda}$ now has an attracting two-cycle that attracts all of the critical points. Hence $F_{\lambda}$ and $F_{-\lambda}=F_{\alpha \lambda}$ are not conjugate.

There is a second natural conjugacy in these families. We have

$$
F_{\bar{\lambda}}(\bar{z})=\bar{z}^{n}+\frac{\bar{\lambda}}{\bar{z}^{d}}=\overline{z^{n}+\frac{\lambda}{z^{d}}}=\overline{F_{\lambda}(z)},
$$

so $F_{\lambda}$ is conjugate to $F_{\bar{\lambda}}$ via $z \mapsto \bar{z}$. Combining these two facts, we have:

Proposition. $F_{\lambda}$ is conjugate to $F_{\mu}$ via

(1) a Möbius transformation if and only if $\mu=\alpha^{2 j} \lambda$ where $\alpha^{n-1}=1$;

(2) an anti-Möbius transformation if and only if $\mu=\alpha^{2 j} \bar{\lambda}$ where $\alpha^{n-1}=1$.

We now turn to the proof of the Escape Time Conjugacy Theorem.

Suppose $\lambda$ belongs to a Sierpiński hole. By a result of McMullen (Corollary 3.6 in [12]; see also [11]), $F_{\lambda}$ is conjugate on its Julia set to a unique critically finite map $F_{\lambda_{0}}$ where $F_{\lambda}$ and $F_{\lambda_{0}}$ lie in the same Sierpiński hole. This proves part (1).

To prove part (2), note that any topological conjugacy $h: J\left(F_{\lambda}\right) \rightarrow$ $J\left(F_{\mu}\right)$ must send $\partial B_{\lambda}$ to $\partial B_{\mu}$, since $B_{\lambda}$ and $B_{\mu}$ are the unique forward- 
invariant Fatou components of $F_{\lambda}$ and $F_{\mu}$. Define the "escape time" of a Fatou component boundary $C$ to be the number of iterations required to reach the boundary of the basin of infinity. The escape time of $C$ and of $h(C)$ are the same, and the escape time $\kappa(\lambda)$ of any map $F_{\lambda}$, defined in terms of critical points, is the same as the escape time of the boundary of the Fatou component containing any critical point $c_{\lambda}$. The set of such boundary curves $C$ surrounding critical points is dynamically distinguished, since these are precisely the boundary curves eventually mapping by degree two onto the boundary of $T_{\lambda}$. Hence, the escape time is a topological conjugacy invariant, proving (2).

We now prove (3). Suppose $F_{\lambda}$ and $F_{\mu}$ are escape time Sierpiński maps which are conjugate on their Julia sets via a homeomorphism $h$. By part (1), we may, and do, assume that these maps are critically finite.

Suppose first that $h$ is orientation preserving. We now modify $h$ off the Julia set to obtain a conjugacy on the whole sphere. As pointed out earlier, the conjugacy $h$ must send $\partial B_{\lambda}$ to $\partial B_{\mu}$. The map $h$ extends to a homeomorphism defined on the entire sphere; see [10].

Let $\Delta$ denote the open unit disk in $\mathbb{C}$. By Böttcher's Theorem, there exist Riemann maps $\phi_{\lambda}, \phi_{\mu}$, unique up to rotation by an $(n-1)$ st root of unity, $\phi_{\lambda}:(\Delta, 0) \rightarrow\left(B_{\lambda}, \infty\right)$ and $\phi_{\mu}:(\Delta, 0) \rightarrow\left(B_{\mu}, \infty\right)$, conjugating $F_{\lambda} \mid B_{\lambda}$ and $F_{\mu} \mid B_{\mu}$ to $z \mapsto z^{n}$. Then $H=\left(\phi_{\mu}\right)^{-1} \circ h \circ \phi_{\lambda}$ conjugates $z^{n}$ on $\partial \Delta$ to itself, so $H(z)=\tau z$ where $\tau^{n-1}=1$. Therefore $H$ extends to a map $H:(\bar{\Delta}, 0) \rightarrow(\bar{\Delta}, 0)$ such that $\phi_{\mu} \circ H \circ \phi_{\lambda}^{-1}$ is an extension of $h$ over $B_{\lambda}$ conjugating $F_{\lambda} \mid B_{\lambda}$ to $F_{\mu} \mid B_{\mu}$. We now assume that $h$ has been so extended. Since $F_{\lambda}$ and $F_{\mu}$ are topologically conjugate on their Julia sets, they are conjugate on the grand orbit of the boundary of the basin of infinity. An easy pullback argument (using induction and the fact that the escape times are the same) shows that for every $N \in \mathbb{N}$, there is a further continuous extension $h_{N}$ of $h$ to a conjugacy such that

$$
h_{N}: J\left(F_{\lambda}\right) \cup \bigcup_{j=0}^{N} F_{\lambda}^{-j}\left(B_{\lambda}\right) \rightarrow J\left(F_{\mu}\right) \cup \bigcup_{j=0}^{N} F_{\mu}^{-j}\left(B_{\mu}\right)
$$

and such that $h_{N}=h_{N-1}$ on the domain of $h_{N-1}$. The sequence of maps $h_{N}$, $N \in \mathbb{N}$, converges since, by local connectivity of the Sierpiński curve, the diameters of the complementary domains must tend to zero. Therefore $F_{\lambda}$ and $F_{\mu}$ are critically finite maps that are topologically conjugate on the entire Riemann sphere. The uniqueness conclusion of Thurston's Theorem (see [9, Theorem 1]) implies that $F_{\lambda}$ and $F_{\mu}$ are Möbius conjugate. Since $h$ must take $\infty$ to $\infty$ and 0 to 0 , the conjugating map must be of the form $h(z)=\beta z$ for some $\beta \in \mathbb{C}$. Then, as we showed in the above Proposition, $\beta$ must be an $(n-1)$ st root of unity and $\mu=\beta^{2 j} \lambda$ for some $j \in \mathbb{Z}$. 
Now suppose that $h$ is orientation reversing. We know that $F_{\lambda}$ and $F_{\bar{\lambda}}$ are conjugate by the orientation reversing map $z \mapsto \bar{z}$ and that $\bar{\lambda}$ is the center of a Sierpiński hole. By the above, the only maps that are conjugate to $F_{\bar{\lambda}}$ via an orientation preserving map are those of the form $F_{\alpha^{2 j} \bar{\lambda}}$. Therefore these are the only maps that can be conjugate to $F_{\lambda}$ by an orientation reversing conjugacy. This completes the proof of the Escape Time Conjugacy Theorem.

3. Conjugacy classes. In this section we compute the number of distinct topological conjugacy classes of escape time Sierpiński curve maps. To do this, it suffices to count the number of centers of the Sierpinski holes that are conjugate either by Möbius transformations or by complex conjugation.

We first assume that $n$ is odd. In this case it is known that there are no Sierpiński holes that meet the real axis in the parameter plane. Indeed, when $\lambda$ is real, only the following situations occur:

1. $v_{\lambda}$ lies in $T_{\lambda}$, so $\lambda$ is in the McMullen domain;

2. $v_{\lambda}$ lies in $B_{\lambda}$, so $\lambda$ lies in the Cantor set locus;

3. the orbit of $v_{\lambda}$ is bounded and $\lambda$ lies in one of the two Mandelbrot sets flanking the origin and straddling the real axis.

See [5] for a proof, and see Figure 2 for a picture of these regions in the parameter plane when $n=3$.

As a consequence, if $\lambda$ lies at the center of a Sierpiński hole, then $\lambda$ cannot be real. Therefore the other centers of Sierpiński holes with conjugate dynamics are those given by parameters of the form $\alpha^{2 j} \lambda$ and $\alpha^{2 j} \bar{\lambda}$ where $j \in \mathbb{Z}$. Since $\alpha$ is an $(n-1)$ st root of unity and $n$ is odd, it follows that there are exactly $n-1$ maps (including $F_{\lambda}$ ) with conjugate dynamics. Thus, by the Structure Theorem, the number of distinct conjugacy classes of escape time $\kappa$ Sierpiński curve maps when $n$ is odd is $(2 n)^{\kappa-3}$.

Now we consider the case where $n$ is even. We shall show that the number of distinct conjugacy classes of escape time Sierpiński curve maps with escape time $\kappa$ is given by $(2 n)^{\kappa-3} / 2+2^{\kappa-4}$. The difference in this case is that there now are centers of the Sierpiński holes lying along the negative real axis in the parameter plane. There are no such holes along the positive real axis since, when $\lambda \in \mathbb{R}^{+}$, we have $F_{\lambda}(x)>0$ when $x>0$. Since $\lambda>0$, there is a critical point on $\mathbb{R}^{+}$. Therefore the critical point on the real axis can never map onto 0 .

Lemma. If $|\lambda| \leq 2$ and $|z| \geq 2$, then $z \in B_{\lambda}$.

Proof. For all $|z| \geq 2$ we have

$$
\left|F_{\lambda}(z)\right| \geq|z|^{n}-\frac{|\lambda|}{|z|^{n}}>|z|^{n}-\frac{2}{|z|^{n}} \geq|z|^{n}-\frac{1}{2^{n-1}}>|z|
$$


since $n \geq 2$. Thus $\left|F_{\lambda}(z)\right|>|z| \geq 2$ and, inductively, $\left|F^{k}(z)\right|>\left|F_{\lambda}^{k-1}(z)\right|$ for all $k$. Hence $F_{\lambda}^{k}(z) \rightarrow \infty$ for all $z$ with $|z| \geq 2$. Therefore all points in the region $|z| \geq 2$ lie in $B_{\lambda}$.

Proposition. If $|\lambda| \geq 2$, then $\lambda$ lies in the escape locus.

Proof. Suppose that $|z| \geq|2 \sqrt{\lambda}|$. Then we have

$$
\left|F_{\lambda}(z)\right| \geq|z|^{n}-\frac{|\lambda|}{|z|^{n}} \geq|z|^{n}-\frac{|\lambda|}{2^{n}|\sqrt{\lambda}|^{n}} \geq|z|^{n}-\frac{1}{2^{n} 2^{n / 2-1}} \geq|z|^{n}-\frac{1}{4}>|z|
$$

since $|z| \geq|2 \sqrt{\lambda}| \geq 2 \sqrt{2}$ and $n \geq 2$. Hence all such $z$ lie in $B_{\lambda}$ and, in particular, $v_{\lambda} \in B_{\lambda}$. Thus, by the Escape Theorem, $\lambda$ lies in the escape locus.

Let us assume for the remainder of this section that $\lambda \notin \mathbb{R}^{+}$and $|\lambda| \leq 2$. Recall that the critical points of $F_{\lambda}$ are given by $c_{\lambda}$ where $c_{\lambda}^{2 n}=\lambda$. Since $\lambda \notin \mathbb{R}^{+}$, there is a unique critical point of $F_{\lambda}$ that lies in the sector $0<$ $\operatorname{Arg} z<\pi / n$. Call this critical point $c_{0}=c_{0}(\lambda)$. Let $c_{j}=c_{j}(\lambda)$ be the critical point given by $\exp (j \pi i / n) c_{0}$, so $c_{2 n-1}$ lies in the sector given by $-\pi / n<\operatorname{Arg} z<0$.

Let $\nu_{j}=\nu_{j}(\lambda)$ denote the ray given by $t c_{j}$ where $t>0$; each $\nu_{j}$ is called a critical point ray. Let $\zeta_{\lambda}^{ \pm}(t)= \pm 2 t \sqrt{\lambda}$ for $t \geq 1$, so the $\zeta_{\lambda}^{ \pm}(t)$ are straight rays that extend from the two critical values $\pm 2 \sqrt{\lambda}$ to $\infty$. We call these rays the critical value rays. Since $F_{\lambda}\left(t c_{\lambda}\right)=\sqrt{\lambda}\left(t^{n}+t^{-n}\right)$ where $t>0$, it follows that $F_{\lambda}$ maps $\nu_{j}(\lambda)$ in two-to-one fashion (except at $c_{j}$ ) over one of the two critical value rays.

Now consider the open region $I_{0}=I_{0}(\lambda)$ defined as follows. By the lemma, the circle $r=4$ lies in $B_{\lambda}$ and, moreover, the critical values lie inside this circle. Hence the preimage of this circle consists of a pair of simple closed curves, $\gamma_{\text {in }}$ and $\gamma_{\text {out }}$, where $\gamma_{\text {in }}$ lies strictly inside $\gamma_{\text {out }}$. Each of these curves is mapped $n$-to- 1 onto $r=4$. Since each of the critical value rays meets $r=4$ in exactly one point, it follows that each of the critical point rays meets $\gamma_{\text {in }}$ and $\gamma_{\text {out }}$ in one point as well.

We let $I_{0}$ be the open region that meets $\mathbb{R}^{+}$and is bounded by the critical point rays $\nu_{0}$ and $\nu_{2 n-1}$ and by the portions of the curves $\gamma_{\text {in }}$ and $\gamma_{\text {out }}$ lying between these rays. Also, let $I_{1}=I_{1}(\lambda)=-I_{0}$. Note that the straight line boundaries of $I_{1}$ lie in $\nu_{n-1}$ and $\nu_{n}$. See Figure 3 .

Proposition. Suppose $|\lambda| \leq 2$ and $\lambda \notin \mathbb{R}^{+}$. Then $F_{\lambda}$ maps $I_{0}$ and $I_{1}$ one-to-one onto a region that contains the set $I_{0} \cup I_{1}$ in its interior.

Proof. Let $v_{\lambda}$ denote the critical value that lies in the upper half-plane. Then, since $0<\operatorname{Arg} \lambda<2 \pi$, we first have

$$
\operatorname{Arg} c_{0}(\lambda)=\frac{1}{2 n} \operatorname{Arg} \lambda<\frac{1}{2} \operatorname{Arg} \lambda=\operatorname{Arg} v_{\lambda} .
$$




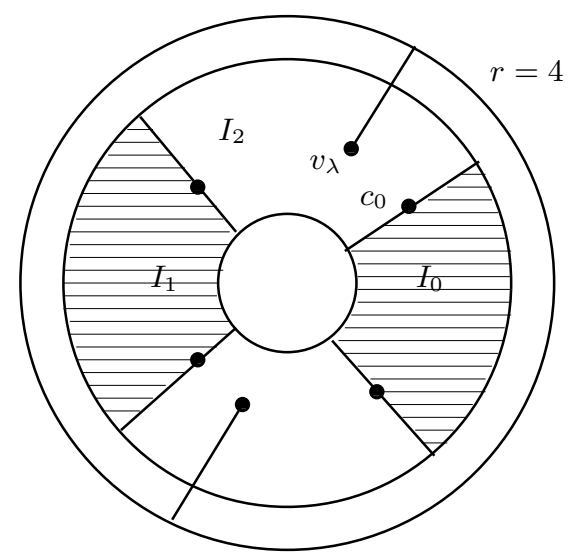

Fig. 3. $I_{0}$ and $I_{1}$ and their image under $F_{\lambda}$, which is the interior of $r=4$ minus the two segments connecting this circle to the critical values. Pictured is the case $n=2$. The region $I_{2}$ will be defined in Section 4 .

We also have

$$
\frac{n-1}{n} \operatorname{Arg} \lambda<\frac{n-1}{n} 2 \pi,
$$

from which it follows that

$$
\operatorname{Arg} v_{\lambda}=\frac{1}{2} \operatorname{Arg} \lambda<\frac{1}{2 n} \operatorname{Arg} \lambda+\frac{n-1}{n} \pi=\operatorname{Arg} c_{n-1}(\lambda) .
$$

As a consequence, the critical value ray in the upper half-plane always lies in the region between the critical point rays $\nu_{0}$ and $\nu_{n-1}$. Similarly, the critical value ray in the lower half-plane always lies between the critical point rays $\nu_{n}$ and $\nu_{2 n-1}$.

Finally, the curve $\gamma_{\text {out }}$ is mapped in one-to-one fashion onto the semicircular portion of $r=4$ that connects the two critical value rays and lies to the right of these rays, while $\gamma_{\text {in }}$ is mapped similarly to the opposite side of $r=4$. Therefore, $F_{\lambda}$ maps $I_{0}$ univalently onto the region $|z|<4$ minus the portions of the critical value rays lying in this disk. Hence $F_{\lambda}\left(I_{0}\right)$ contains both $I_{0}(\lambda)$ and $I_{1}(\lambda)$ in its interior as long as $\lambda \notin \mathbb{R}^{+}$and $|\lambda| \leq 2$. By symmetry, the same is true for $F_{\lambda}\left(I_{1}\right)$.

As a consequence of this Proposition, there are unique points in $I_{0}$ and $I_{1}$ that are mapped to 0 by $F_{\lambda}$. Furthermore, there is an open set $I_{00} \subset I_{0}$ having the property that $F_{\lambda}$ maps $I_{00}$ univalently onto $I_{0}$ and another set $I_{01} \subset I_{0}$ that is mapped univalently onto $I_{1}$. Similarly, there are subsets $I_{10}$ and $I_{11}$ of $I_{1}$ that are mapped univalently onto $I_{0}$ and $I_{1}$ respectively. Hence there is a unique point in each of these four sets that is mapped to 0 by $F_{\lambda}^{2}$. Inductively, given a sequence $S$ of $j+10$ 's and 1's, $S=s_{0} s_{1} \ldots s_{j}$, there is a subset $I_{s_{0} \ldots s_{j}}$ of $I_{s_{0}}$ that is mapped univalently onto $I_{s_{j}}$ by $F_{\lambda}^{j}$. Hence 
there is a unique point in this set that is mapped to 0 by $F_{\lambda}^{j+1}$. We call this point $x_{\lambda}^{S}$. Note that the orbit of $x_{\lambda}^{S}$ passes through the $I_{s_{k}}$ in the exact order determined by the sequence $S$ before landing on 0 .

REMARK. Standard arguments from complex dynamics can be used to show that there is an $F_{\lambda}$-invariant Cantor set contained inside $I_{0} \cup I_{1}$. This is the beginning of the construction of Cantor necklaces in both the dynamical and the parameter planes (see [7]). However, we will not make use of this concept in this paper.

Our goal now is to prove that, given any finite sequence $S=s_{0} \ldots s_{j}$, there is a unique parameter $\lambda_{S} \in \mathbb{R}^{-}$for which the critical orbits all land on $x_{\lambda_{S}}^{S}$ at the second iteration, and hence $F_{\lambda_{S}}^{j+3}\left(c_{\lambda_{S}}\right)=0$. It turns out that the proof of this when $n=2$ is very different from the cases where $n$ is greater than 2. One reason for this is the absence of a McMullen domain in the parameter plane when $n=2$, but there are other reasons that we will discuss in the next section. Therefore, for the remainder of this section, we will further assume that $n \geq 4$ (and even).

Proposition. Suppose $n \geq 4$ is even. If $|\lambda| \leq 0.01$, then $\lambda$ lies in the McMullen domain.

Proof. Recall that the McMullen domain is an open disk that surrounds the origin in the parameter plane. Recall also that the critical values are given by $v_{\lambda}= \pm 2 \sqrt{\lambda}$. If $|\lambda| \leq 0.01$, then

$$
\begin{aligned}
\left|F_{\lambda}( \pm 2 \sqrt{\lambda})\right| & \geq \frac{|\lambda|}{2^{n}|\sqrt{\lambda}|^{n}}-2^{n}|\sqrt{\lambda}|^{n} \geq \frac{1}{2^{n}|\lambda|^{(n-2) / 2}}-(0.2)^{n} \\
& \geq \frac{1}{2^{n}(0.1)^{n-2}}-2^{n}(0.1)^{n}=\frac{5^{n-2}}{4}-(0.2)^{n}>2 .
\end{aligned}
$$

By the Lemma, for each $\lambda$ with $|\lambda| \leq 0.01, F_{\lambda}\left(v_{\lambda}\right)$ lies in $B_{\lambda}$. We claim that $v_{\lambda}$ lies in $T_{\lambda}$. To see this, let $z$ lie on the circle of radius $|\lambda|^{1 / 2 n}$ centered at the origin. Then

$$
\left|F_{\lambda}(z)\right| \leq 2|\lambda|^{1 / 2}<|\lambda|^{1 / 2 n}
$$

since $|\lambda| \leq 0.01$. Hence the circle of radius $|\lambda|^{1 / 2 n}$ is mapped strictly inside itself and so this circle does not lie in $B_{\lambda}$. Moreover, $v_{\lambda}$ lies inside this circle. It then follows that $v_{\lambda}$ must lie in $T_{\lambda}$. By the Escape Theorem, each $\lambda$ with $0<|\lambda| \leq 0.01$ therefore lies in the McMullen domain.

Still assuming that $n \geq 4$ is even, let $\mathcal{W}$ denote the set of parameters that satisfy

1. $(n-2) \pi /(n-1)<\operatorname{Arg} \lambda<n \pi /(n-1)$;

2. $0.01<|\lambda|<2$ 


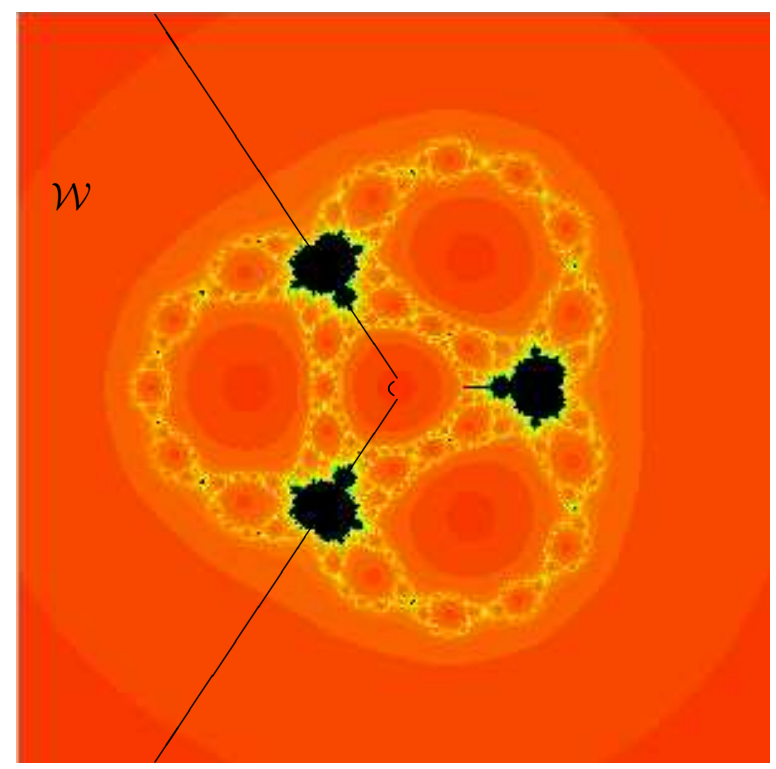

Fig. 4. The region $\mathcal{W}$ in the parameter plane when $n=4$

(see Figure 4). We shall henceforth restrict our attention to parameters in $\mathcal{W}$ (note that $\mathcal{W}$ contains the portion of $\mathbb{R}^{-}$between -2 and -0.01 ). Let $G: \mathcal{W} \rightarrow \mathbb{C}$ be given by

$$
G(\lambda)=F_{\lambda}^{2}\left(c_{\lambda}\right)=2^{n} \lambda^{n / 2}+\frac{1}{2^{n} \lambda^{(n-2) / 2}} .
$$

Note that, since $n$ is even, all of the critical points of $F_{\lambda}$ land on the same point after two iterations of $F_{\lambda}$, so $G(\lambda)$ is well defined and analytic on $\mathcal{W}$. Also, define

$$
\mathcal{I}=\bigcup_{\lambda \in \mathcal{W}}\left(I_{0}(\lambda) \cup I_{1}(\lambda)\right) .
$$

Proposition. If $n \geq 4$ is even, the map $G$ takes $\mathcal{W}$ univalently onto an open set that completely contains the closure of $\mathcal{I}$.

Proof. A computation shows that $G$ has $n-1$ critical points given by

$$
\left(\frac{n-2}{4^{n} n}\right)^{1 /(n-1)}
$$

One of these critical points lies on the straight ray whose argument is given by $(n-2) \pi /(n-1)$. Call this critical point $\xi$. There is another critical point, $\bar{\xi}$, on the ray given by $\operatorname{Arg} \lambda=n \pi /(n-1)$. Note that these critical points lie on the straight line boundaries of $\mathcal{W}$. We call the ray given by $\operatorname{Arg} \lambda=(n-2) \pi /(n-1)$ the upper ray bounding $\mathcal{W}$, while the ray given by 
$\operatorname{Arg} \lambda=n \pi /(n-1)$ is the lower ray bounding $\mathcal{W}$. Then another computation shows that the upper ray bounding $\mathcal{W}$ is mapped by $G$ to the ray with argument

$$
-\frac{\pi}{2(n-1)}+\frac{n-1}{2} \pi
$$

while the lower ray bounding $\mathcal{W}$ is mapped to the ray with argument

$$
\frac{\pi}{2(n-1)}+\frac{n+1}{2} \pi \text {. }
$$

Indeed, $G$ maps the upper ray two-to-one (except at $\xi$ ) onto the portion of its image ray extending from $G(\xi)$ to $\infty$, and $G$ maps the lower ray in similar fashion onto the portion of its image ray extending from $G(\bar{\xi})$ to $\infty$.

Note that the arguments of these two image rays sum to $n \pi$ so these image rays are the negatives of each other. Let $X$ be the pair of sectors in $\mathbb{C}$ given by

$$
\frac{\pi}{2}-\frac{\pi}{2(n-1)} \leq|\operatorname{Arg} z| \leq \frac{\pi}{2}+\frac{\pi}{2(n-1)} .
$$

Then, by the above, the images under $G$ of the upper and lower bounding rays of $\mathcal{W}$ lie on the boundaries of $X$.

Now recall that $I_{0}(\lambda)$ is bounded by the critical point rays $\nu_{0}(\lambda)$ and $\nu_{2 n-1}(\lambda)$. Therefore $I_{0}$ is contained in the region

But we have

$$
-\frac{\pi}{n}<\frac{1}{2 n} \operatorname{Arg} \lambda-\frac{\pi}{2 n}<\operatorname{Arg} z<\frac{1}{2 n} \operatorname{Arg} \lambda<\frac{\pi}{n} .
$$

$$
\frac{\pi}{2}-\frac{\pi}{2(n-1)}>\frac{\pi}{n} \quad \text { and } \quad-\frac{\pi}{2}+\frac{\pi}{2(n-1)}<-\frac{\pi}{n},
$$

so it follows that the images of the upper and lower bounding rays of $\mathcal{W}$ are a bounded distance away from $\bigcup_{\lambda} I_{0}(\lambda)$. In a similar fashion, these images are bounded away from $\bigcup_{\lambda} I_{1}(\lambda)$ as well.

Finally, since $G(\lambda) \approx 2^{n} \lambda^{n / 2}$ on the circle $|\lambda|=2$, it follows easily that $G$ maps this portion of the boundary of $\mathcal{W}$ one-to-one to a nearly circular curve that lies well outside the circle of radius 2 and connects the images of the upper and lower bounding rays in the portion of the plane to the right of these rays. Similarly, $G$ maps the circular boundary given by $|\lambda|=0.01$ onto a nearly circular curve that again lies outside the circle of radius 2 and also connects the images of the upper and lower bounding rays, but this time in the region to the left of these rays. As a consequence, $G$ maps $\mathcal{W}$ univalently over a set that strictly contains the closure of $\mathcal{I}$.

Note that the above result implies that there is a unique parameter $\lambda_{0} \in \mathcal{W}$ for which $G\left(\lambda_{0}\right)=0$, i.e., $\lambda_{0}$ is the center of a Sierpiński hole with escape time 3 . In fact, solving $G(\lambda)=0$ shows that there are $n-1$ such centers of Sierpiński holes in $\mathbb{C}$, namely those given by $\lambda^{n-1}=-1 / 4^{n}$. Here, 
$\lambda_{0}$ is the center of the Sierpiński hole that lies in $\mathbb{R}^{-}$, and all of these other maps are conjugate to $F_{\lambda_{0}}$ via a Möbius transformation of the form $z \mapsto \alpha^{j} z$ where $\alpha^{n-1}=1$.

Proposition. For each itinerary $S=s_{0} \ldots s_{j}$, there is a unique parameter $\lambda^{S}$ for which each of the critical points lands on $x_{\lambda}^{S}$ after two iterations. Moreover, $\lambda^{S} \in \mathbb{R}^{-}$.

Proof. Fix an itinerary $S=s_{0} \ldots s_{j}$. We have two maps defined on $\mathcal{W}$. The first is the map $\lambda \mapsto x_{\lambda}^{S}$ and the second is $\lambda \mapsto G(\lambda)$. Both of these maps are analytic functions defined on $\mathcal{W}$. Moreover, $G$ is invertible and $G(\mathcal{W})$ contains the closure of $\mathcal{I}$, so we can consider the map $P: \mathcal{W} \rightarrow \mathcal{W}$ given by $P(\lambda)=G^{-1}\left(x_{\lambda}^{S}\right)$. Since $x_{\lambda}^{S}$ lies in a compact subset of $G(\mathcal{W})$, it follows from the Schwarz Lemma that $P$ has a unique fixed point $\lambda_{S}$ in $\mathcal{W}$. This fixed point is a parameter for which $G\left(\lambda_{S}\right)=x_{\lambda_{S}}^{S}$, i.e., $F_{\lambda_{S}}^{k+3}\left(c_{\lambda_{S}}\right)=0$ and the second iterate of $c_{\lambda_{S}}$ is a point that travels around $I_{0}$ and $I_{1}$ with the required itinerary before landing on 0 .

We claim that $\lambda_{S} \in \mathbb{R}^{-}$. To see this, note first that $G(\lambda)$ is real when $\lambda \in \mathbb{R}^{-}$. A straightforward computation then shows that $G$ takes the interval $[-2,-0.01)$ in one-to-one fashion onto a line that contains the interval $[-2,2]$ in its interior. Now when $\lambda$ is real, $x_{\lambda}^{S}$ is also real. Hence the map $G^{-1}\left(x_{\lambda}^{S}\right)$ must have at least one fixed point in $[-2,0.01]$. But, from the above, we know that this must then be the unique fixed point of $P$.

We now compute the number of centers of Sierpiński holes that have conjugate dynamics when $n>2$ is even. For each itinerary $s_{0} \ldots s_{k-1}$, there is a unique $\lambda \in \mathbb{R}^{-}$for which the critical orbits land on 0 at iteration $k+2$, so $\lambda$ is the center of a Sierpiński hole of escape time $k+3$. These are the only real parameters with this property.

Since $n$ is even, there are a total of $n-1$ conjugate parameters for each of these maps, so we have exactly $2^{k}(n-1)$ parameters that are in conjugacy classes containing a real parameter. Thus the number of parameters that do not have this property is $(2 n)^{k}(n-1)-2^{k}(n-1)$. Each of these maps is conjugate to exactly $2(n-1)$ other such maps, including itself, so there are $(2 n)^{k} / 2-2^{k-1}$ such "complex" conjugacy classes and $2^{k}$ "real" conjugacy classes. Altogether, this gives

$$
(2 n)^{k} / 2+2^{k-1}
$$

conjugacy classes of maps with escape time $k+3$, or equivalently,

$$
(2 n)^{\kappa-3} / 2+2^{\kappa-4}
$$

conjugacy classes with escape time $\kappa$. 
4. The case $n=2$. We finally turn to the case where $n=2$, i.e., to the map

$$
F_{\lambda}(z)=z^{2}+\frac{\lambda}{z^{2}} .
$$

One difference between this case and the previous ones is that there is no McMullen domain in the parameter plane for these maps. Indeed, we have $G(\lambda)=F_{\lambda}\left(v_{\lambda}\right)=4 \lambda+1 / 4$ so $F_{\lambda}\left(v_{\lambda}\right) \rightarrow 1 / 4$ as $\lambda \rightarrow 0$ (as opposed to $F_{\lambda}\left(v_{\lambda}\right) \rightarrow \infty$ when $\left.n \geq 3\right)$. Therefore $v_{\lambda}$ never lies in $T_{\lambda}$ in this case. In fact, it is known that, in any neighborhood of the origin in the $\lambda$-plane, there are infinitely many different Sierpiński holes (see [3]). See Figure 5 for a magnification of the parameter plane near $\lambda=0$.

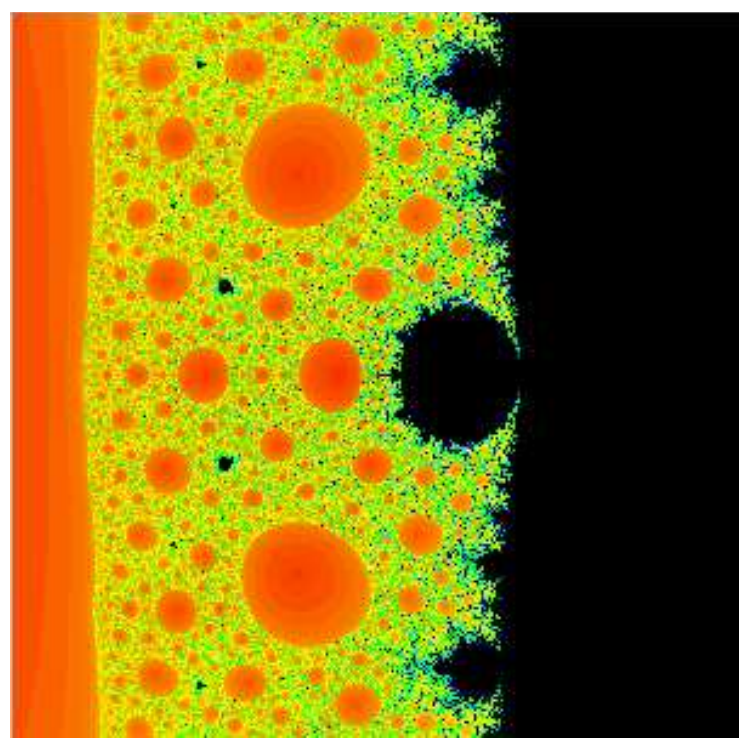

Fig. 5. The region $-0.03 \leq x, y \leq 0.03$ in the parameter plane when $n=2$. The origin is at the tip of the "tail" of the distorted Mandelbrot set on the right.

In this case we may still define the regions $I_{0}(\lambda)$ and $I_{1}(\lambda)$, and we have $F_{\lambda}\left(I_{j}\right) \supset I_{0} \cup I_{1}$ as before. Since there is no McMullen domain, the region $\mathcal{W}$ in the parameter plane must now extend to 0 , so we now let

$$
\mathcal{W}=\{\lambda|0<| \lambda \mid<2,0<\operatorname{Arg} \lambda<2 \pi\} .
$$

The major problem that arises when attempting to count the number of different conjugacy classes is that, since $G(\lambda)=4 \lambda+1 / 4, G(\mathcal{W})$ no longer properly contains $I_{0}(\lambda)$. However, $G(\mathcal{W})$ is the disk of radius 8 about $1 / 4$ minus the line segment $[0.25,8.25]$ in $\mathbb{R}^{+}$, so we do have $G(\mathcal{W}) \supset I_{1}(\lambda)$ for each $\lambda \in \mathcal{W}$. Hence, if $S=s_{0} \ldots s_{k-1}$ is an itinerary for which $s_{0}=1$, then the above proof shows that there is a unique $\lambda_{S}$ as before. 
Let $S=s_{0} \ldots s_{k-1}$ where $s_{0}=0$. Let $I_{2}=I_{2}(\lambda)$ be the region in the dynamical plane bounded by the curves $\gamma_{\text {in }}$ and $\gamma_{\text {out }}$ and also lying in the sector

$$
\frac{1}{2 n} \operatorname{Arg} \lambda=\operatorname{Arg} c_{0}<\operatorname{Arg} z<\operatorname{Arg} c_{1}=\frac{1}{2 n} \operatorname{Arg} \lambda+\frac{\pi}{2},
$$

so $I_{2}(\lambda)$ is an open region lying between $I_{0}$ and $I_{1}$ in the upper half plane (see Figure 3).

Note that $F_{\lambda}$ takes $I_{2}$ one-to-one onto the exact same region as $F_{\lambda}$ maps $I_{0}$ and $I_{1}$. Hence $F_{\lambda}\left(I_{2}\right) \supset I_{0} \cup I_{1}$ for each $\lambda$. Thus there is a unique point $p_{\lambda}^{S}$ lying in $I_{2}$ and having the property that $F_{\lambda}\left(p_{\lambda}^{S}\right)=x_{\lambda}^{S}$ for each $\lambda \in \mathcal{W}$. Thus $F_{\lambda}^{k+1}\left(p_{\lambda}^{S}\right)=0$.

Instead of the map $G$, let us now consider the map $H: \mathcal{W} \rightarrow \mathbb{C}$ given by $H(\lambda)=2 \sqrt{\lambda}$ where $\operatorname{Im} \sqrt{\lambda}>0$, so $H(\lambda)$ is the critical value of $F_{\lambda}$ that lies in the upper half-plane. As in the case of $G$, the map $H$ is analytic and univalent on $\mathcal{W}$.

Proposition. $H(\mathcal{W})$ contains $I_{2}(\lambda)$ for each $\lambda \in \mathcal{W}$.

Proof. Since $I_{2}(\lambda)$ and $H(\mathcal{W})$ are contained in the upper half-plane, we need only show that $H$ maps the outer boundary of $\mathcal{W}$ outside $\gamma_{\text {out }}$. To see this, suppose that $|\lambda|=2$ so that $\left|v_{\lambda}\right|=2 \sqrt{2}$. If $|z|=2 \sqrt{2}$, then we have

$$
\left|F_{\lambda}(z)\right| \geq|z|^{2}-\frac{|\lambda|}{|z|^{2}}=8-\frac{1}{4}>4 \text {. }
$$

Consequently, $F_{\lambda}(z)$ lies outside $r=4$. Therefore $z$ lies outside $\gamma_{\text {out }}$ and so the image of the outer boundary of $\mathcal{W}$ under $H$ lies outside $\gamma_{\text {out }}$.

We claim that there is a unique parameter $\lambda_{S}$ in $\mathcal{W}$ such that $H\left(\lambda_{S}\right)$ $=p_{\lambda}^{S}$. As before, we shall show that $\lambda \mapsto H^{-1}\left(p_{\lambda}^{S}\right)$ has a unique fixed point $\lambda_{S}$ in $\mathcal{W}$ and that $\lambda_{S} \in \mathbb{R}^{-}$. The problem here is that, unlike the previous case, $H(\mathcal{W})$ does not properly contain the closure of $\bigcup_{\lambda}\left(I_{2}(\lambda)\right)$, so it is not immediately clear that this map has a fixed point in $\mathcal{W}$.

To show that there is indeed a fixed point, we return to the map $G$. Fix an itinerary $s_{0} \ldots s_{k-1}$ that begins with a certain number of 0 's, say $s_{0}=\cdots=s_{j}=0$ with $0<j \leq k-1$, but either $s_{j+1}=1$ or else $j=k-1$. As in the previous case, we can produce an interval $I_{s_{0} \ldots s_{k-1}}$ whose points move through the interval $(0,2] \subset \mathbb{R}^{+}$in the prescribed fashion before being mapped at iteration $k$ over all of $[-2,2]$. But when $\lambda \in[-2,0), G(\lambda)$ only covers $[-7.75,0.25)$. However, for $\lambda$ close to $0, F_{\lambda}$ is close to $x \mapsto x^{2}$ except in some small neighborhood of 0 . Under $x \mapsto x^{2}$, any point close to $x=1 / 4$ remains in $(0,1 / 4]$ for all iterations. Hence, given any $\varepsilon>0$, we may choose $\lambda \in \mathbb{R}^{-}$small enough so that every point in the interval $(1 / 4-\varepsilon, 1 / 4)$ has itinerary that begins with $s_{0}=\cdots=s_{j+1}=0$ under each $F_{\lambda}$. That is, each point in this interval remains in $\mathbb{R}^{+}$for at least $j+1$ iterations. By 
choosing $\lambda$ even smaller, we may assume that $G(\lambda)$ also lies in the interval $(1 / 4-\varepsilon, 1 / 4)$.

Now consider the point $x_{\lambda}^{S}$ as defined earlier. This point has itinerary that begins with only $j+10$ 's. The $(j+1)$ st entry in the itinerary may be 1 or the point may land on the origin at the corresponding iteration, but $s_{j+1} \neq 0$. Hence $x_{\lambda}^{S}$ does not lie in the interval $(1 / 4-\varepsilon, 1 / 4)$. Therefore $x_{\lambda}^{S}$ lies to the left of $G(\lambda)$ when $\lambda$ is close enough to 0 . But when $\lambda<-1 / 16$, we have $G(\lambda)<0$, so $x_{\lambda}^{S}$ lies to the right of $G(\lambda)$. Hence, by continuity, there must be at least one parameter for which $G(\lambda)=x_{\lambda}^{S}$. For this parameter, we therefore have $H(\lambda)=p_{\lambda}^{S}$ where $F_{\lambda}\left(p_{\lambda}^{S}\right)=x_{\lambda}^{S}$. This then produces a fixed point for the map $H^{-1}\left(p_{\lambda}^{S}\right)$. The rest of the proof then follows immediately from the Schwarz Lemma.

\section{References}

[1] M. Bonk, Quasiconformal geometry of fractals, in: International Congress of Mathematicians, Vol. II, Eur. Math. Soc., 2006, 1349-1373.

[2] M. Bonk, B. Kleiner and S. Merenkov, Rigidity of Schottky sets, Amer. J. Math., to appear.

[3] R. L. Devaney, A myriad of Sierpiński curve Julia sets, in: Difference Equations, Special Functions and Orthogonal Polynomials, World Sci., 2007, 131-148.

[4] - The McMullen domain: satellite Mandelbrot sets and Sierpiński holes, Conformal Geom. Dynamics 11 (2007), 164-190.

[5] —, Baby mandelbrot sets adorned with halos in families of rational maps, in: Complex Dynamics (Twenty-Five Years After the Appearance of the Mandelbrot Set), Contemp. Math. 396, Amer. Math. Soc., 2006, 37-50.

[6] - Structure of the McMullen domain in the parameter planes for rational maps, Fund. Math. 185 (2005), 267-285.

[7] - Cantor necklaces and structurally unstable Sierpinski curve Julia sets for rational maps, Qualit. Theory Dynam. Systems 5 (2004), 337-359.

[8] R. L. Devaney, D. M. Look and D. Uminsky, The escape trichotomy for singularly perturbed rational maps, Indiana Univ. Math. J. 54 (2005), 1621-1634.

[9] A. Douady and J. Hubbard, A proof of Thurston's topological characterization of rational functions, Acta Math. 171 (1993), 263-297.

[10] J. Krasinkiewicz, On homeomorphisms of the Sierpinski curve, Prace Mat. (Comment Math.) 12 (1969), 255-257.

[11] R. Mañé, P. Sad and D. Sullivan, On the dynamics of rational maps, Ann. Sci. École Norm. Sup. 16 (1983), 193-217.

[12] C. McMullen, Automorphisms of rational maps, in: Holomorphic Functions and Moduli, Vol. 1, Math. Sci. Res. Inst. Publ. 10, Springer, New York, 1988, 31-60.

[13] J. Milnor and Tan Lei, A "Sierpiński carpet" as Julia set, Appendix F in Geometry and dynamics of quadratic rational maps, Experiment. Math. 2 (1993), 37-83.

[14] P. Roesch, On capture zones for the family $f_{\lambda}(z)=z^{2}+\lambda / z^{2}$, in: Dynamics on the Riemann Sphere, Eur. Math. Soc., 2006, 121-129.

[15] N. Steinmetz, On the dynamics of the McMullen family $R(z)=z^{m}+\lambda / z^{\ell}$, Conformal Geom. Dynamics 10 (2006), 159-183. 
[16] G. T. Whyburn, Topological characterization of the Sierpinski curve, Fund. Math. 45 (1958), 320-324.

Department of Mathematics

Department of Mathematics

Boston University

Indiana University

Boston, MA 02215, U.S.A.

E-mail: bob@bu.edu

Received 20 May 2008;

in revised form 2 September 2008 